perceptron is identical to the threshold logic gate. Consequently, all properties satisfied by a threshold gate will also apply to binary perceptrons. According to its definition, a threshold gate or a binary perceptron having $n$ inputs $x_{n}$, $\mathbf{x}_{n-1}, \ldots, x_{1}$ with $\mathrm{n}$ weights $w_{n}, w_{n-1}, \ldots, w_{1}$ on the corresponding input lines or connections, realises a Boolean function $\mathbf{F}$, such that

$$
\begin{aligned}
F & =1 & \text { when } \sum_{i=1}^{n} w_{i} x_{i} \geq T \\
& =0 & \text { when } \sum_{i=1} w_{j} x_{i}<t
\end{aligned}
$$

where the $w_{i} s, T$, and $t$ are real numbers, and $\mathbf{x}$, can assume only one of the two values 0 and 1 . The quantity $\sum w_{i} x_{i}$ is called the weighted sum and $\mathrm{T}$ and $t$ are the upper and lower bound, respectively, of the threshold. In our case the weights are always integers (both positive and negative), and therefore the gap between the upper and lower bound of threshold is always 1 , i.e. $T-t=1$. The actual value of threshold $\theta$ is fixed at the middle of the gap. Hence $8=T-0 \cdot 5$. As is well known, the Boolean function realisable by a single perceptron is a linearly separable (LS) function. ${ }^{3,4}$ The realisation vector (RV) comprising the weight vector (WV) and the threshold $\theta$ of an LS function separates the true vertices from the false vertices of an n-dimensional hypercube. In the terminology of switching theory, it separates the on minterms whose outputs are $1 \mathrm{~s}$ from the of minterms whose outputs are $0 \mathrm{~s}$.

A minterm is a product term of $n$ variables. It is known'" that all product terms are LS functions. Its realisation vector is given by the following theorem. A parameter of minterms, which we shall require is defined below.

Definition $I$ : The number of $\mathbf{I s}$ in a minterm expressed in its binary form, where the complemented and the true forms of a variable are denoted by 0 and 1 respectively, is known as its rowsum (RS).

Theorem I: An n-variable minterm $b_{n}, b_{n-1}, \ldots, b_{1}$ can be separated from the rest of the minterms by weight vector $w$, $w_{n-1}, \ldots, w_{1}$, where $w_{i}$ is given by

$$
\begin{aligned}
w_{i} & =1 & & \text { if } b_{i}=1 \\
& =-1 & & \text { if } b_{i}=0
\end{aligned}
$$

The threshold 8 is given by $\theta=M-0.5$, where $M$ is the rowsum of the minterm.

Theorem 1 describes a well known result, and its proof can be found in references 3 and 4 .

Theorem 2: Tuning theorem: The realisation vector (w: 0 ) realising the single n-variable minterm $m$, will also realise all minterms having Hamming distance $d$ or less from the minterm $m_{k}$ if only the threshold $\theta$ in the realisation vector is reduced by $d$, that is, it is made $\theta-d$, the weight vector $\mathbf{w}$ remaining unaltered.

\title{
TUNING CAPABILITY OF BINARY \\ PERCEPTRON
}

Indexing term: Neural networks

A theorem called the tuning theorem, which brings out the tuning capability of perceptrons where both the inputs and outputs are binary, has been stated and proved. It has been shown how with the help of this property, an associative memory has been developed with a single layer of percepmemory has been developed withel a singe layer of percepa fully connected iterative Hopfield network.

In a perceptron as defined by Rosenblatt, ${ }^{1}$ and Minsky and Papert ${ }^{2}$ the output is binary but the input may be binary or continuous. If we restrict the inputs to be only binary valued, we obtain what may be called a binary perceptron. Such a

Proof: Let the minterm $m_{k}$ have $1 \mathrm{~s}$ at bit positions $b_{i}$ and $0 s$ at bit positions $b_{j}$. Therefore by theorem 1 , in its realisation vector the corresponding $\boldsymbol{w}_{i} s$ are $1 \mathrm{~s}$ and $\boldsymbol{w}_{j} \mathrm{~s}$ are $-1 \mathrm{~s}$. The weighted sum of $m_{\mathbf{k}}$ is $\sum \mathrm{w},=\sigma_{\mathrm{k}}$ say. Now, let a new minterm $m_{p}$ having a Hamming distance of only 1 from $m_{k}$, be obtained from $m$, by changing one of its $b_{i} s$ into 0 . Therefore, the weighted sum of the new minterm $\boldsymbol{m}_{p}$ will be $\sigma_{p}=\sigma_{k}-\boldsymbol{w}_{i}=$ $\sigma_{k}-1$. Again let a new minterm $m_{q}$ that has a Hamming distance of only 1 from $m_{k}$ be obtained from $m_{k}$ by changing one of its $b_{j} \mathrm{~s}$ into 1 . The weighted sum of the new minterm $m_{\mathrm{g}}$ will thus be $\sigma_{q}=\sigma_{k}+w_{j}=\sigma_{k}-1$.

Therefore, irrespective of how the new minterm of Hamming distance 1 is obtained, its weighted sum will be $\sigma_{k}-1$. This minterm can be realised by reducing the threshold $\theta$ by 1 . Extending the argument it can be seen that the weighted sum of a minterm that has a Hamming distance $d$ from $m_{k}$, can be realised by reducing $\theta$ by $d$. Obviously, such a reduced threshold will also realise all minterms having Hamming distance less than $d$. 
It is now evident that a perceptron acts as a filter whose selectivity can be tuned to accept all minterms up to a Hamming distance $\boldsymbol{d}$ by adjusting its threshold. Taking this significant property and also the coding capability offered by heorem 1 as the basis, we have developed an associative memory (AM) with binary perceptrons. The AM is to recognise the correct identity of a pattern corrupted by binary noise. It stores the patterns of $\mathbf{2 6}$ alphabets represented on a $9 \times 9$ grid as has been done by Michel et al. ${ }^{5}$ We use 26 neurons (binary perceptrons) each having 81 weights to code each of the alphabets. Note that the 81 bit code word of an alphabet can be thought of as a minterm of an 81-variable Boolean function. It can therefore be stored with 81 weights Boolean function. It can the fore be AM has 81 inputshts of B neuron as given by the sponding to each bit. The 81 inpus are connected in a paralle alphabet. When a noisy pattern comes at the input, each neuron simultaneously computes its weighted sum. Each weighted sum at a neuron falls short by $d$ from its threshold, where $\boldsymbol{d}$ is the Hamming distance of the input pattern from the stored pattern. The stored pattern has been fixed as its weights once for all when the neuron learnt the code of the alphabet. Now, a controller network connected from, and to, all the individual neurons decreases the threshold of all the neurons simultaneously in steps of 1 . As soon as the decreneurons sime decrewich is obviously closest to the patten fires. The fining of a which is obviously closest to the pattern fires. The firing of weuron inhibits the controller net patrom decreasing the thresholds further. We have simulated the working of the AM When the AM is fed with the noisy input pattern of $\mathrm{M}$ a shown in Fig. 1b (taken from Fig. 3 of Reference 5) the neuron M fires. For neuron M, the $\mathrm{d}$ is 19 , whereas the $d s$ for other neurons having very similar pattern such as $\mathrm{N}, \mathrm{W}$ and $\mathrm{H}$ were $\mathbf{2 2 ,} 27$ and 26, respectively.

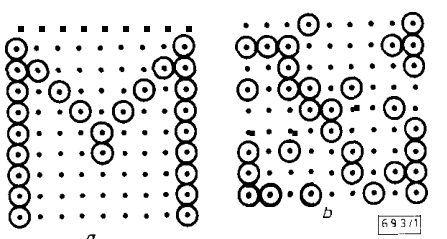

Fig. 1 Stored pattern and noisy (binary) input pattern ofalphabet $M$ in $9 \times 9$ grid

$a$ Stored pattern

$b$ Noisy (binary) input pattern

The AM as developed by us has many advantages ove those of Michel et al. s $^{5}$ and many other similar models which are variations of the Hopfield network. ${ }^{6}$ The learning which amounts to the fixing of weights according to theorem 1 is very simple. Any number of characters can be stored, the very simple. Any n. The ger neviden eferes only the number of connections to each neuron, rather than increasing the number of neurons. All stored patterns ar stable, and the AM is guaranteed to recognise the closest pattern. In case the noisy pattern is equidistant from more than one stored character, more than one neuron indicating the situation fire simultaneously.
References

1 ROSENBLATT, F.: 'The perceptron: A probabilistic model for information storage and organi

2 MINSKY, M, and PAPERT, S.: 'Perceptrons' (Cambridge, MA, MIT Press, 1988), expanded edition

3 LEWIS, P. M., and COATES, C. L.: 'Threshold logic' (New York, Wiley, 1967)

4 BISWAS, N. N.: 'Introduction lo logic and switching theory' (New York, Gordon and Breach, 1975)

5 MICHEL, A. N., SI, J., and YEN, G.: 'Analysis and synthesis of a clas of discrete-time neural networks described on hypercubes', IEE Trans., 1991, N-2, (1), pp. 32-46

6 HOPFIELD, J. J.. Neural networks and physical systems with emergent collective computational abilities'. Proc. National Academy 